\title{
Bi-objective supply chain problem using MOPSO and NSGA-II
}

\author{
Hassan Javanshir $^{\mathrm{a}^{*}}$, Sadoullah Ebrahimnejad ${ }^{\mathrm{b}}$ and Samaneh Nouri ${ }^{\mathrm{a}}$
}

${ }^{a}$ Department of Industrial Engineering, Islamic Azad University, South Tehran Branch, Iran ${ }^{b}$ Department of Industrial Engineering, Islamic Azad University, Karaj Branch, Iran

\section{A B S T R A C T}

\begin{abstract}
The increase competition and decline economy has increased the relevant importance of having responsiveness supply chain. This has created much motivations to reduce the cost of services and, at the same time, to increase the quality of services. In this paper, we present a multi-stage supply chain network by considering multi products, single resource and deterministic cost and demand. There are two objectives of minimizing total cost and maximizing total responsiveness, which is the minimization of total numbers of delayed products and services. The proposed model of this paper is formulated as a mixed integer linear programming and we present two metaheuristics namely MOPSO and NSGA-II to solve the resulted problems. The performance of the proposed models of this paper has been examined using some randomly generated numbers and the results are discussed. The preliminary results indicate that while MOPSO is able to generate more Pareto solutions in relatively less amount of time, NSGA-II is capable of providing better quality results.
\end{abstract}

\section{Introduction}

The increase competition and decline economy has increased the relevant importance of having reliable supply chain and there have been tremendous efforts on providing efficient supply chain solutions. A supply chain consists of a set of suppliers, storages or distribution centers (DSs) and customers. In other words, SC is an integrated system for managing systems' components, activities and information for serving products or services to different customers. The objective is to minimize total costs associated with the system and to meet all customers' requirements (Papageorgiou, 2009; Tuzkaya \& Onut, 2009).

Designing a network is one of the most important strategic decision making where we determine the number, location and capacity of facilities and the amount of materials which are used in production operations or distributed among facilities. Such decisions strongly influence on final costs and customers' services (Altiparmak et al., 2006).

\footnotetext{
* Corresponding author. Tel: +98 2177508894

E-mail: h_javanshir@azad.ac.ir (H. Javanshir)

(C) 2012 Growing Science Ltd. All rights reserved. doi: $10.5267 /$ j.. jiec.2012.02.003
} 
There is normally more than one single objective associated with network design and most of these objectives are in conflict with each other. In fact, the objective is to find a solution, which compromise among different solutions (Pishvaee et al., 2010).

One important issue in many studies is to find effective method for locating efficient solutions. There are literally various criteria for measuring the relative efficiencies of solutions in terms of qualitative and quantitative factors. In qualitative, we cannot precisely assign a value to a particular criterion and examples of such criteria include customer satisfaction, integration of flow and information, effective risk management, suppliers' performance and quality of products. On the contrary, quantitative criteria such as minimization of cost, maximization of return of investment, minimization of delivery data, etc. can be measured, precisely (Beamon, 1998).

Customers normally evaluate services based on their perception from what they receive or expect to receive. Therefore, the gap between what customers expect and what they receive is a multi factors item and in many cases, customers are satisfied when they receive more than what they expect (Bottani \& Rizzi, 2006). In this article, we proposed a mixed integer linear programming (MILP) to design an integrated multi stages supply chain with multi products and single resource. The problem is categorized as NP-Hard and we propose some metaheuristics and to solve the resulted bi-objective problem.

The organization of this paper first presents literature review in section 2 and section 3 introduces the proposed model of the paper. Section 4 provides details of some experimental test and section 5 discusses details of our findings. Finally, concluding remarks are given in the last to summarize the contribution of the paper.

\section{Literature review}

During the past two decades, there have been tremendous efforts on supply chain planning. Voudouris and consulting (1996) are believed to be the first pioneers who presented mathematical programming techniques to debottleneck the supply chain of fine chemicals. The model was based on a discrete time representation to capture the dynamics of the system, and it can be utilized to simulate an existing scheduling policy and evaluated its efficiency. Beamon (1998) is believed to be one of the first who provided a comprehensive review of literature in multi-stage supply chain modeling and defined a research agenda for future research in this area.

Sabri and Beamon (2000) presented a multi-objective technique for simultaneous strategic and operational planning in supply chain design. The model considered production, delivery, and demand uncertainty, and provided a multi-objective performance vector for the entire SC network. You and Grossmann (2008) also investigated design of responsive supply chain under demand uncertainty.

Lee and Kim (2002) presented an analytic technique to solve the integrated production-distribution problems in supply chain management (SCM). They explained there are many real-world applications where there are various types of uncertain factors such as unexpected delays, queuing, etc. and analytic model cannot handle the problem, precisely. They proposed a hybrid method by integrating the analytic and simulation model, where the operation time in the analytic model was considered as a dynamic factor and it was adjusted by the results from independently developed simulation model.

Perea-López et al. (2003) presented a model predictive control strategy for supply chain optimization to maximize profit in supply chains with multi-product, multi-echelon distribution networks with multiproduct batch plants. Their proposed model considers a discrete time mixed integer linear programming dynamic model, which considers the flow of material and information within the system. The proposed model also considers a general framework, which considers all the elements of the supply chain and their interactions. The study compared the behavior of a supply chain under different centralized and decentralized approaches, and demonstrated that the former had better results. 
Erol and Ferrell (2004) presented a technique to support decision making across the supply chain of an industrial distributor, which simultaneously resolves the two fundamental decisions facing a distributor including assigning suppliers to warehouses and warehouses to customers and they also investigated two important issues. First, they considered their model in multi-objective form since distributors frequently must concurrently meet various conflicting objectives such as minimization of cost and customer satisfaction. They also considered qualitative and quantitative parameters involve with their problem formulation.

Gen and Syarif (2005) presented a hybrid genetic algorithm for multi-time period production/distribution planning. They considered a production/distribution problem, which determines integration of production, distribution and inventory system so that products are produced and distributed in an appropriate quantities, to suitable customers, on time by minimizing system wide costs while satisfying all demand required. This problem is considered as an optimization model, which integrates distribution costs, facility location decisions, and inventory management for multi-products and multi-time periods. To solve the problem, they proposed a spanning tree-based genetic algorithm (hst-GA) and it was hybridized with the fuzzy logic controller (FLC) concept for auto-tuning the GA parameters. GA has been widely used for many real-world supply chain applications. Zanjirani Farahani and Elahipanah (2008), for instance, presented a GA to optimize the total cost and service level for just-in-time distribution in a supply chain. Zhou et al. (2002) used GA method for balanced allocation of customers to multiple distribution centers in the supply chain network.

There are other metaheuristics approaches used for supply chain applications. Particle swarm optimization is one of the most popular approaches used in the literature (CoelloCoello et al., 2004; CoelloCoello et al., 2007; Poli et al., 2007; Das et al., 2008; Reyes-Sierra \& CoelloCoello, 2006; Guliashki et al., 2009; Sedighizadeh \& Masehian, 2009; Sharaf \& El.Gammal, 2009). Guillén et al. (2005) considered the design and retrofit issue of a supply chain (SC), which is in several production plants, warehouses and markets, and the associated distribution systems. Their approach was capable to consider and manage the financial risk associated to the various design options, resulting in a set of Pareto optimal solutions, which could be implemented for decision-making.

Altiparmak et al. (2006) proposed a technique based on genetic algorithms (GAs) to detect a set of Pareto-optimal solutions for multi-objective supply chain network (SCN) design problem. They presented two various weight techniques to handle the nature of multi-objective and enabled the decision maker to find a greater number of alternative solutions. They used the proposed model of their paper for a real-world case study of plastic products in Turkey. The proposed solution procedure and simulated annealing were compared according to quality of Pareto-optimal solutions to assess the performance of the proposed model.

Bottani and Rizzi (2006) explained that logistics and supply chain management that customer service management plays strategic role for firms in coming years by improving logistics performances, companies increase customer satisfaction and gain market shares. They introduced a technique for the management of customer service based on the quality function deployment (QFD) and addressed the issue of how to deploy the house of quality (HOQ) to improve logistics processes and provide more customer satisfaction. The methodology has been tested by means of a real case application, which refers to an Italian company operating in the mechanical industry. Xu et al. (2008) presented a class of multi-objective supply chain network optimal model under random fuzzy environment and its application to the industry of Chinese Liquor. Altiparmak et al. (2009) presented a new method for multi-product supply chain network design using a steady state genetic algorithm (ssGA). Their proposed model uses a new encoding structure for the design of a single-source, multi-product, multistage SCN. They evaluated the performance of their proposed model with the results obtained by CPLEX, Lagrangean heuristic, hyrid GA and simulated annealing on a set of SCN design problems with various sizes. 
Cardona-Valdés et al. (2010) introduced a bi-objective supply chain problem where all parameters are under uncertainty. They considered the design of a two-echelon production distribution network by considering various manufacturing plants, customers and a set of candidate distribution centers. The primary contribution of the study was to extend the existing literature by considering the demand uncertainty of customers within the distribution center location and transportation decisions. They also provided a network design, which satisfies economical and service quality objectives of the decision maker within two levels supply network setting. The proposed model was then formulated in two-stage integer recourse problem to find Pareto optimal network configuration and assignment of transportation modes and the respective flows to optimize total cost and service time, simultaneously. They also presented a stochastic optimization technique under demand uncertainty, where the inherent risk was considered by various scenarios. Finally, they proposed solution procedure for the proposed stochastic optimization problem based on L-shaped algorithm within an -optimality structure and validated their model using numerical results.

Papageorgiou (2009) studied a critical review of methodologies for enhancing the decision-making for process industry supply chains. He explained that the existing uncertainty within supply chains is an important issue for efficient capacity utilization and robust infrastructure decisions. Chan and Kumar (2009) provided a new technique for effective allocation of customers to distribution centers using a multiple ant colony optimization approach. They considered a multiple ant colony optimization (MACO) technique to design a balanced and efficient supply chain network, which maintains the best balance of transit time and customers service. They determined the effective allocation of the customers to distribution centers (DCs) by considering two objectives of the transit time and degree of imbalance of the DCs and used MACO technique in the context of ant colony system, where multiple ant colonies cooperate with each other to locate the best possible customer allocation pattern for the DC.

Reverse logistics, induced by different forms of return, has become of interesting subject of research throughout this decade and reverse logistics network design (RLND) is one of the main important strategic issues. Du and Evans (2008) performed an analysis of RLND, which deals with the returns requiring repair service. In their proposed model, a problem consists of a manufacturer outsourcing to a third-party logistics (3PLs) provider for its post-sale services was investigated. The proposed model is a bi-objective optimization model and both objectives, which are minimization of the overall costs and total tardiness of cycle time were investigated. The primary objective of the research was to determine a set of non-dominated solutions to the facility capacity arrangement along with the associated transportation flows between customer areas and service facilities using three algorithms including scatter search, the dual simplex method and the constraint method. Their numerical solutions also indicated that the optimization for the first objective function led to a centralized network structure and the minimization of the second objective function yielded in a decentralized network structure.

Tuzkaya and Önüt (2009) presented a holonic approach based integration methodology for transportation and warehousing functions of the supply network. Pishvaee et al. (2010) presented a memetic algorithm for bi-objective integrated forward/reverse logistics network design. They studied a model for integrated logistics network design to prevent the sub-optimality caused by a separate, sequential design of forward and reverse logistics networks. The proposed model was formulated as multi-objective mixed integer programming, which minimizes the total costs and maximizes the responsiveness of a logistics network. Since the resulted problem formulation was NP-Hard, they presented a multi-objective memetic algorithm by employing three different local searches. They also compared their Pareto-optimal solutions with other existing techniques in the literature.

The main difference between the Altiparmak et al.'s works (2009) with the proposed model of this paper is associated with the second objective function and the proposed strategy for solving the resulted problem formulation. 


\section{The proposed model}

The proposed model of this paper considers a multi-level supply chain network by considering multi products, single resource and deterministic cost and demand. In other words, there are different factories, distribution centers and multiple numbers of customers. There are two objectives associated with the proposed model of this paper: the first objective function considers total cost of supply chain while the second objective function reduces delays in serving customers. The decision variables determine the number of production units as well as the number of distribution centers to meet customers' requirements. In this problem, we make the necessary decisions on a subset of factories and DCs, which must be kept open. We also determine the amount of production in each factory and the amount of goods transferred between different facilities. The main strategy in this production/distribution network is to meet customers' demand by considering all related capacity limitations as well as maintaining total costs and lateness. The following assumptions hold for the proposed model of this paper,

1. There are predefined numbers of factories, distribution centers with known capacities.

2. The number of customers and their demands along with their capacities are known.

3. Any customer receives its demand only from one distribution center.

4. Customers are categorized in three different levels. The first level is associated with important customers, $\gamma_{1}$, whose complains must be considered seriously. The complain for the second category of customers, $\gamma_{2}$, are relatively important. The last group of customers, $\gamma_{3}$, are those whose complain are not very important.

In this paper, those customers with demand ratio in $[75,100]$ are considered in the first category with $\alpha=\frac{3}{6}$, people with demand ratio in $[35,75]$ are considered in the second group with $\alpha=\frac{2}{6}$ and the last group is associated with people whose demand is in $[10,35]$ with $\alpha=\frac{1}{6}$.

\subsection{Notations}

i: Set of customers with $i \in I$ $\mathrm{j}$ : Set of distribution centers $j \in J$

\subsection{Parameters}

$g_{k} \quad$ Fixed operating cost of factory $k$

$o_{j} \quad$ Fixed operating cost of distribution center $j$

$v_{l k} \quad$ Production cost for each item $l$ in factory $k$

$s_{s k r} \quad$ Transportation cost of each unit of raw material

$a_{k j l} \quad$ Transportation cost each unit final product $l$ from factory $k$ to distribution center $j$

$c_{j i l} \quad$ Transportation cost each unit final product $l$ from distribution center $j$ to customer $i$

$d_{i l} \quad$ Demand of product $l$ for customer $i$

$\alpha_{i l} \quad$ Relative importance of customer $i$ for product $l$

$w_{j} \quad$ Annual capacity of distribution center $j$

$W \quad$ Maximum number of distribution centers

$D_{k} \quad$ Capacity of factory $k$

$P \quad$ Maximum number of factories

$n_{l} \quad$ Rate of required space for product $l$ in a particular distribution center 
$m_{l} \quad$ Utilization rate of each production unit $l$

$u_{r l} \quad$ Utilization rate of raw material $r$ in each production unit $l$

$s p_{s r} \quad$ Capacity ratio of supplier $s$ for raw material $r$

$t_{l i} \quad$ Due date of product $l$ for customer $i$

$t_{l j i} \quad$ The amount of time required to deliver product $l$ from distribution center $j$ to customer $i$

$\vartheta_{j} \quad$ The cost of each unit capacity for each distribution center in location $j$

\subsection{Decision makers}

$z_{j}=\left\{\begin{array}{cc}1 & \text { if distribution center } j \text { is open } \\ 0 & \text { otherwise }\end{array} \quad p_{k}=\left\{\begin{array}{cc}1 & \text { if factory } \mathrm{k} \text { is open } \\ 0 & \text { otherwise }\end{array}\right.\right.$

$$
y_{j i}=\left\{\begin{array}{lc}
1 & \text { if distribution center } j \text { serves customer } i \\
0 & \text { otherwise }
\end{array}\right.
$$

$b_{s k r}$ : the amount of raw material $r$ shipped from supplier $s$ to factory $k$

$x_{l k}$ : the amount of product $l$ in factory $k$

$q_{j i l}$ : the amount of product $l$ shipped from distribution center $j$ to customer $i$

$f_{k j l}$ : the amount of product $l$ shipped from factory $k$ to distribution $j$

\subsection{The proposed model}

The proposed model of this paper is as follows,

$$
\begin{aligned}
\min Z_{1}= & \sum_{j} o_{j} z_{j}+\sum_{k} g_{k} p_{k}+\sum_{l} \sum_{k} v_{l k} x_{l k}+\sum_{s} \sum_{k} \sum_{r} t_{s k r} b_{s k r}+ \\
& \sum_{k} \sum_{j} \sum_{l} a_{k j l} f_{k j l}+\sum_{j} \sum_{i} \sum_{k} c_{j i l} q_{j i l}+\sum_{j} \sum_{i} \sum_{l} \vartheta_{j} d_{i l} y_{j i} \\
\min Z_{2}= & \sum_{l} \sum_{i} \sum_{j} \max \left[\left(t_{l j i}-t_{l i}\right), 0\right] \cdot q_{j i l} \cdot \alpha_{i l}
\end{aligned}
$$

subject to

$\sum_{j} y_{j i}=1$,

$\sum_{i} \sum_{l} n_{l} d_{i l} y_{j i} \leq w_{j} z_{j}, \quad \forall j$

$\sum_{j} z_{j} \leq W$,

$q_{j i l}=d_{i l} y_{j i}, \quad \forall i, j, l$

$\sum_{k} f_{k j l}=\sum_{i} q_{j i l}, \quad \forall j, l$

$\sum_{k} b_{s k r} \leq s p_{s r}, \quad \forall s, r$

$\sum_{k} p_{k} \leq P$,

$\sum_{j} f_{k j l} \leq x_{l k}, \quad \forall k, l$

$\sum_{l} u_{r l} x_{l k} \leq \sum_{s} b_{s k r}, \quad \forall r, k$

$\sum_{l} m_{l} x_{l k} \leq D_{k} P_{k}, \quad \forall k$ 


$$
\begin{array}{lc}
z_{j}=\{0,1\}, & \forall j \\
p_{k}=\{0,1\}, & \forall k \\
y_{j i}=\{0,1\}, & \forall i, j \\
b_{s k r} \geq 0, & \forall s, k, r \\
x_{l k} \geq 0, & \forall l, k \\
q_{j i l} \geq 0, & \forall i, j, l \\
f_{k j l} \geq 0 . & \forall k, j, l
\end{array}
$$

The first objective function considers fixed costs associated with distribution centers, production units, variable costs of production and distribution centers and transportation cost between facilities, which are based on number of units carried and the distance between various units. The second objective function minimizes any delays in delivery of products to customers. Constraint sets (3) determine the allocation of distribution centers to customers. Constraint sets (4) determine capacity of distribution centers. Constraint sets (5) determine the number of open facilities. Constraints (6) and (7) represent customer satisfaction and demands for DCs while constraint (8) shows the limitation on raw material. Constraint (9) determines the number of open factories. Constraint (10) indicates that the number production units shipped from a particular factory cannot be more than its limitation. Constraint (11) determines the amount of necessary raw materials required and constraints (12) shows the capacity of factory. Constraints (13)-(19) indicate that the variables are positive and some of them are binary variables.

\section{Solution procedure}

In the proposed model of this paper, there are two conflicting objectives and we need to generate some Pareto optimal solutions defined as follows,

$\min \vec{f}(\vec{x})=\left\{f_{1}(\vec{x}), \cdots, f_{k}(\vec{x})\right\}$

$g_{i}(\vec{x}) \leq 0, i=1, \cdots, M$,

$h_{i}(\vec{x})=0, i=1, \cdots, P$,

$\vec{x} \in S$,

where $\vec{x}=\left[\mathrm{x}_{1}, \mathrm{x}_{2}, \ldots, \mathrm{x}_{\mathrm{n}}\right]^{\mathrm{T}}$ are decision variables, and $\mathrm{f}_{\mathrm{i}}: \mathrm{R}^{\mathrm{n}} \rightarrow \mathrm{R} \mathrm{i}=1, \ldots, \mathrm{k}$ are objective functions. In addition $g_{i}, h_{j}: R^{n} \rightarrow R, i=1, \ldots, m, j=1, \ldots, p$ are inequality and equality constrains, respectively. Decision variable $\overrightarrow{x_{1}} \in S$ dominates $\overrightarrow{x_{2}} \in S$ if the values of objective functions with $\overrightarrow{x_{1}}$ will not be worse than the objective functions on $\overrightarrow{x_{2}}$ and, at least, it is better in one simple case. $\overrightarrow{x_{1}} \in S$ is Pareto optimal if there is no other solution $\overrightarrow{\mathrm{x}_{2}} \in \mathrm{S}$, which yields better solution. In multi-objective solution, it is normally impossible to have a solution where one objective function always performs better than the others since the objectives are in serious conflict.

\subsection{Particle Swarm Optimization (PSO)}

Particle swarm optimization (PSO) has been one of the most popular techniques, which can be used and it is efficient when compared with mathematical methods. PSO incorporates particles or population of individuals to move through the $d$-dimensional search space and each individual keeps a speed, which acts as an operator to obtain a new set of individuals. The population of candidate solutions in PSO technique is transferred through the search space updating the positions according to velocity factors. The principle is based on the idea that each solution can be represented as a particle in a swarm and each particle has a position and velocity vector. Each position coordinate shows a parameter value. In a 
physical $d$-dimensional search space, the position and velocity of individual $i$ are represented as the vectors $P_{i}=\left(p_{i 1}, \cdots, p_{i d}\right) \quad$ and $V_{i}=\left(v_{i 1}, \cdots, v_{i d}\right)$, respectively. Let Pbest $t_{i}=\left(p_{i 1}^{\text {Pbest }}, \cdots, p_{i d}^{\text {Pbest }}\right)$, and Gbest $_{i}=\left(p_{i 1}^{\text {Gbest }}, \cdots, p_{i d}^{\text {Gbest }}\right)$ be the best location of individual $i$ and its neighbors' best location, respectively. Using the information, the updated velocity of individual $i$ is modified under the following equation in the PSO method:

$$
\begin{gathered}
V_{i}^{k+1}(t+1)=w \times V_{i}^{k}(t)+c_{1} \times \operatorname{rand}() \times\left(\text { Pbest }_{i}^{k}(t)-P_{i}^{k}(t)\right) \\
+c_{2} \times \operatorname{rand}() \times\left(\text { Gbest }_{i}^{k}(t)-P_{i}^{k}(t)\right), \\
P_{i}^{k+1}(t+1)=P_{i}^{k}(t)+V_{i}^{k+1}(t+1),
\end{gathered}
$$

where $w$ is the inertia weighting factor, $c_{1}$ and $c_{2}$ are weight factors; and rand() is random number between 0 and 1. For more details, please see Chen and Wang (2010).

\subsubsection{Multi objective PSO (MOPSO)}

On the contrary to PSO, multi-objective PSO (MOPSO) there is no gbest and one of the best nondominated solutions is considered. The possibility of choosing a particular solution has a reverse relationship with the population. Details of the proposed model is described in CoelloCoello et al. (2004) and for sake of simplicity they are repeated in this paper. For the proposed model of this paper, we consider the initial population as $100, w=0.15, c_{1}=0.35, c_{2}=0.5, \alpha=0.1, \beta=3, \gamma=2$. The number of iterations is also set to 100 .

\subsection{Sorting genetic algorithm non-dominated-II (NSGA-II)}

The proposed sorting genetic algorithm no dominated-II (NSGA-II) was first introduced by Deb et al. (2002) and maintains a complexity of $\mathrm{O}\left(\mathrm{MN}^{2}\right)$. As we explained earlier, there are two objective functions of cost and delivery time associated with the proposed model of this paper. Therefore, we provide a set of so-called Pareto optimal solutions using the proposed meta-heuristic approaches. In the first step of NSGA-II algorithm, an initial population P0 is generated, randomly. In each generation $t$, the following processes are carried out. All the offspring chromosomes Qt, the population of children, are created with operations namely selection, crossover and mutation and they are evaluated.

Then, all the individuals from Pt and Qt are ranked and they are placed in varying fronts. First Pareto front which is not dominated by other front is constituted and includes all the non dominated solutions. In order to find the solutions in the next front, only the remaining solutions are considered. We repeat this process until ranking of all solutions are carried out and they are assigned to several fronts. After that, the best solutions, in the best front and with the most crowding distance, are selected for the new population $\mathrm{Pt}+1$. This generation is stopped if the stopping criterion is satisfied. The overall structure of the NSGA-II is demonstrated in the following subsection.

\subsubsection{Structure of the NSGA-II}

Create the initial population $\mathrm{P} 0$ of size $\mathrm{N}$

Estimate generated solutions

Rank these solutions by non domination and sort them by crowding distance

While stopping criterion is not verified do

Generate the offspring population Qt by selection, crossover and mutation

Constitute the populations of parents and the children in $\mathrm{Rt}=\mathrm{Pt} \mathrm{U} \mathrm{Qt}$

Sort the solutions of new population Rt in different Pareto fronts Fi by the Pareto dominance

$\mathrm{Pt}+1=0$ 
$\mathrm{i}=1$

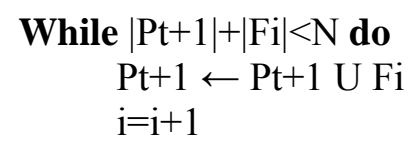

\section{End while}

Include in $\mathrm{Pt}+1, \mathrm{~N}-|\mathrm{Pt}+1|$ solutions of Fi by descending order of the crowding distance

\section{End while}

In this paper, to solve NSGA-II, the number of population is set to 100 , termination criterion was set to 100 and the crossover ratio was set to 1 with the possibility of 0.3 . Since the problem is continuous crossover to increase diversity of the resulted solutions and $\alpha$ is set from 0.15 to 1.15 . Hence, we set mutation probability equal to 0.5 and a mutation rate equal to 0.1 . For mutation and crossover operations, we have used competitive method.

\subsection{Penalty functions}

Both NSGA-II and MOPSO only optimize the raw variables and for each main variable, one raw variable in a region of zero to one is defined. Then using a mathematical approach all raw materials are mapped on original variables to calculate the objective function. In the event the constraints are not met we add a penalty in the objective function.

There are seven types of variables associated with the proposed model of this paper and we need to define $\hat{Z}_{j} \in[0,1]$ is converted to $Z_{j} \in\{0,1\}$ so that the constraint $\sum_{j} Z_{j} \leq W$ holds. We first sort all members of $\widehat{Z}_{j}$ in non-decreasing order and all components with the value greater than 0.5 are set to one and the rest of them are set to zero keeping in mind the number of component does not violate $\sum_{\mathrm{j}} \mathrm{Z}_{\mathrm{j}} \leq$ W. We repeat the same argument for $\mathrm{P}_{\mathrm{k}}$ by considering $\sum_{\mathrm{k}} \mathrm{p}_{\mathrm{k}} \leq \mathrm{P}$. To handle $\mathrm{y}_{\mathrm{ji}}$ for converting $\hat{y}_{j \mathrm{i}} \in[0,1]$ to $\mathrm{y}_{\mathrm{ji}} \in\{0,1\}$ we need to keep in mind that when $\mathrm{Z}_{\mathrm{j}}=0$ then $\mathrm{y}_{\mathrm{ji}}=0$. Therefore, $\hat{\mathrm{y}}_{\mathrm{ji}}$ needs to be considered as $Z_{\mathrm{j}} \cdot \hat{y}_{\mathrm{ji}}$. Since each distributer has to serve one single customer, i.e. $\sum_{\mathrm{j}} \mathrm{y}_{\mathrm{ji}}=1$, we rank distributers and choose the one with the highest rank. In constraint, $\sum_{\mathrm{i}} \sum_{\mathrm{l}} \mathrm{n}_{\mathrm{l}} \mathrm{d}_{\mathrm{il}} \mathrm{y}_{\mathrm{ji}} \leq \mathrm{W}_{\mathrm{j}} \mathrm{Z}_{\mathrm{j}}$, when $\sum_{\mathrm{i}} \sum_{\mathrm{l}} \mathrm{n}_{\mathrm{l}} \mathrm{d}_{\mathrm{il}} \mathrm{y}_{\mathrm{ji}}$ is considered for $\mathrm{DUC}_{\mathrm{j}}$ and $\mathrm{W}_{\mathrm{j}} \mathrm{Z}_{\mathrm{j}}$ is considered for $\mathrm{DAC}_{\mathrm{j}}$ then $\mathrm{DUC}_{\mathrm{j}} \leq \mathrm{DAC}_{\mathrm{j}}$ must hold, otherwise we consider a penalty as follows,

$D C V_{j}=\max \left(\frac{D U C_{j}}{D A C_{j}}-1.0\right)$.

In Eq. (26), there is no penalty when constraint holds and when it does not we consider a positive penalty. We use similar approach for $\sum_{\mathrm{k}} \mathrm{b}_{\mathrm{skr}} \leq \mathrm{sp}_{\mathrm{sr}}$ and $\sum_{l} \mathrm{~m}_{\mathrm{l}} \mathrm{x}_{\mathrm{lk}} \leq \mathrm{D}_{\mathrm{k}} \mathrm{P}_{\mathrm{k}}$. In terms of $\mathrm{f}_{\mathrm{kjl}}$ to convert $\hat{\mathrm{f}}_{\mathrm{kjl}} \in[0,1]$ into the original variable, if $\mathrm{P}_{\mathrm{k}}$ is zero then $\mathrm{f}_{\mathrm{kjl}}$ will be zero. In addition, since $\sum_{\mathrm{k}} \mathrm{f}_{\mathrm{kjl}}=$ $\mathrm{Q}_{\mathrm{jl}}=\sum_{\mathrm{i}} \mathrm{q}_{\mathrm{jil}}, \mathrm{f}_{\mathrm{kjl}}$ is calculated as follows,

$f_{k j l}=\frac{\hat{f}_{k j l} P_{k} Q_{j l}}{\sum_{k \dot{o}} \hat{f}_{\dot{o} j l} P_{k \dot{o}}}$.

Since $\mathrm{x}_{\mathrm{lk}}$ depends on $\mathrm{f}_{\mathrm{kjl}}$, i.e. $\sum_{\mathrm{j}} \mathrm{f}_{\mathrm{kjl}}=\mathrm{x}_{\mathrm{lk}}$, we can calculate $\mathrm{x}_{\mathrm{lk}}$ based on $\mathrm{f}_{\mathrm{kjl}}$. In constraint $\sum_{\mathrm{l}} \mathrm{m}_{\mathrm{l}} \mathrm{x}_{\mathrm{lk}} \leq$ $\mathrm{D}_{\mathrm{k}} \mathrm{P}_{\mathrm{k}}$, let $\mathrm{PUC}_{\mathrm{k}}=\sum_{\mathrm{l}} \mathrm{m}_{\mathrm{l}} \mathrm{x}_{\mathrm{lk}}$ and $\mathrm{PAC}_{\mathrm{k}}=\mathrm{D}_{\mathrm{k}} \mathrm{P}_{\mathrm{k}}$. Therefore, we must have $\mathrm{PUC}_{\mathrm{k}} \leq \mathrm{PAC}_{\mathrm{k}}$. Again we consider a penalty as follows,

$P C V_{j}=\max \left(\frac{P U C_{j}}{P A C_{j}}-1.0\right)$.

In order to change $\hat{b}_{\text {skr }} \in[0,1]$ into $b_{\text {skr }}$ note that when $P_{k}=0$ then $b_{\text {skr }}=0$. Since we have $B_{k r}=$ $\sum_{\mathrm{l}} \mathrm{u}_{\mathrm{rl}} \mathrm{x}_{\mathrm{lk}} \leq \sum_{\mathrm{s}} \mathrm{b}_{\mathrm{skr}}$ then we must have, 
$b_{s k r}=\frac{\hat{b}_{s k r} P_{k} B_{k r}}{\sum_{s \dot{b}} \hat{b}_{s \dot{o} k r} P_{k \dot{o}}}$,

Let SUC $=\sum_{\mathrm{k}} \mathrm{b}_{\mathrm{skr}}$ and $\mathrm{SAC}_{\mathrm{s}}=\mathrm{sp}_{\mathrm{sr}}$, in order to have $\mathrm{SUC}_{\mathrm{s}} \leq \mathrm{SAC}_{\mathrm{s}}$ we consider the following penalty,

$S C V_{s}=\max \left(\frac{S U C_{s}}{S A C_{s}}-1\right)$,

The average penalty function is added to the objective function as follows,

$$
\tilde{Z}=Z+P(\bar{V})
$$

or $\tilde{Z}=\mathrm{Z}(1+\beta \overline{\mathrm{V}})$ where $\beta$ is a positive parameter, which is $\beta=5$ in this paper.

\section{Results}

\subsection{Quality of solutions}

In this paper, we use different criteria to measure the performance of the algorithms such as the number Pareto optimal solutions, quality of Pareto optimal solutions, CPU times, etc.

\subsubsection{Uniformly distribution of Pareto optimal solutions}

This is the first and somehow important index, which shows how close the Pareto optimal solutions are and it is as follows,

$$
S=\sqrt{\frac{1}{n-1} \sum_{i=1}^{n}\left(\bar{d}-d_{i}\right)^{2}},
$$

where $n$ is the number of Pareto optimal solutions, $d_{i}$ is the distance to $i^{\text {th }}$ closest optimal solution, which is calculated as follows,

$$
d_{i}=\min _{j}\left(\left|f_{1}^{i}(x)-f_{1}^{j}(x)\right|+\left|f_{2}^{i}(x)-f_{2}^{j}(x)\right|\right) .
$$

It is clear that smaller values of $\mathrm{S}$ represent better values.

\subsubsection{Quality of solution}

Let $\mathrm{A}$ and $\mathrm{B}$ be the quality of the solutions obtained by two algorithms. $\mathrm{C}(\mathrm{A}, \mathrm{B})$ is defined as the ratio, which is calculated as follows,

$C(A, B)=\frac{\text { Number of solutions B dminated by Solutions } \mathrm{A}}{\text { Number of solutions B }}$

Similarly we have,

$C(B, A)=\frac{\text { Number of solutions A dminated by Solutions B }}{\text { Number of solutions B }}$,

and we have,

$Q(A, B)=\frac{C(A, B)}{C(A, B)+C(B, A)}$,

$Q(B, A)=\frac{C(B, A)}{C(A, B)+C(B, A)}$,

$\mathrm{Q}(\mathrm{A}, \mathrm{B})+\mathrm{Q}(\mathrm{B}, \mathrm{A})=1$.

It is obvious that better quality solutions are more desirable. 


\subsubsection{Diversity of Pareto optimal solutions}

The other criteria is the diversity of the solutions, which can be calculated as follows,

$$
D=\sum_{k=1}^{n_{o b j}} \max _{i, j}\left|f_{k}(i)-f_{k}(j)\right| \text {. }
$$

In Eq. (39), $i$ and $j$ are the index of solutions and $k$ is the index of the objective function, respectively.

\subsection{The results}

We have considered 16 sample problems and ran two algorithms five different times in different sizes. The parameters of the proposed model have been adjusted in Table 1 as follows,

Table 1

The input parameters of the proposed model

\begin{tabular}{cccccc}
\hline$g_{\mathrm{k}}$ & $o_{\mathrm{j}}$ & $v_{\mathrm{lk}}$ & $t_{\mathrm{skr}}$ & $a_{\mathrm{kjl}}$ & $c_{\mathrm{jil}}$ \\
$u[50000,150000]$ & $u[10000,30000]$ & $u[1,10]$ & $u[12000,240000]$ & $u[10000,50000]$ & $u[10000,50000]$ \\
$d_{\mathrm{il}}$ & $\propto_{\mathrm{il}}$ & $\mathrm{w}_{\mathrm{j}}$ & $\mathrm{W}, P$ & $D_{\mathrm{k}}$ & $\vartheta_{j}$ \\
$u[10,100]$ & $u[0,1]$ & $u[3000,10000]$ & 100 & $u[3000,10000]$ & $u[1,10]$ \\
$n_{\mathrm{l}}$ & $m_{\mathrm{l}}$ & $u_{\mathrm{rl}}$ & $s p_{\mathrm{sr}}$ & $t_{\mathrm{li}}$ & $t_{\mathrm{lji}}$ \\
$u[0.2 .5]$ & $u[0.2 .5]$ & $u[0.2 .5]$ & $u[3000,25000]$ & $u[1,25]$ & $u[1,75]$ \\
\hline
\end{tabular}

Table 2

The input parameters for some sample small (S) and large (L) problems

\begin{tabular}{|c|c|c|c|c|c|c|c|c|c|c|c|c|c|}
\hline & I & $\mathrm{J}$ & K & $\mathrm{S}$ & $\mathrm{L}$ & $\mathrm{R}$ & & I & $\mathrm{J}$ & $\mathrm{K}$ & $\mathrm{S}$ & $\mathrm{L}$ & $\mathrm{R}$ \\
\hline S1 & 10 & 5 & 5 & 2 & 2 & 2 & L1 & 100 & 20 & 10 & 2 & 2 & 2 \\
\hline S2 & 10 & 5 & 5 & 2 & 3 & 2 & L2 & 100 & 30 & 15 & 2 & 3 & 2 \\
\hline S3 & 20 & 5 & 5 & 2 & 2 & 2 & L3 & 200 & 30 & 15 & 2 & 2 & 2 \\
\hline S4 & 20 & 10 & 5 & 2 & 3 & 3 & L4 & 200 & 40 & 20 & 2 & 3 & 2 \\
\hline S5 & 30 & 5 & 5 & 2 & 2 & 2 & L5 & 300 & 40 & 20 & 2 & 2 & 2 \\
\hline S6 & 30 & 10 & 5 & 2 & 3 & 2 & L6 & 300 & 50 & 25 & 2 & 3 & 3 \\
\hline S7 & 40 & 10 & 5 & 2 & 2 & 2 & & & & & & & \\
\hline S8 & 40 & 15 & 10 & 2 & 3 & 2 & & & & & & & \\
\hline S9 & 50 & 10 & 5 & 2 & 2 & 2 & & & & & & & \\
\hline $\mathrm{S} 10$ & 50 & 15 & 10 & 2 & 3 & 2 & & & & & & & \\
\hline
\end{tabular}

As we can observe from Fig. 1, MOPSO mostly provides more Pareto optimal solutions compared with NSGA-II algorithm.

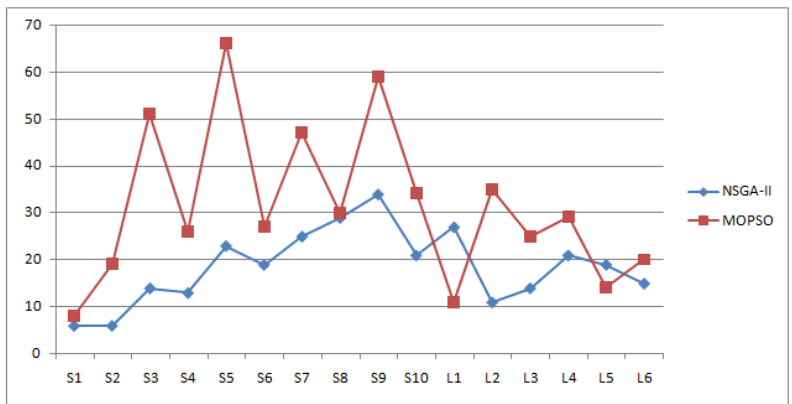

Fig. 1. The number of Pareto solutions

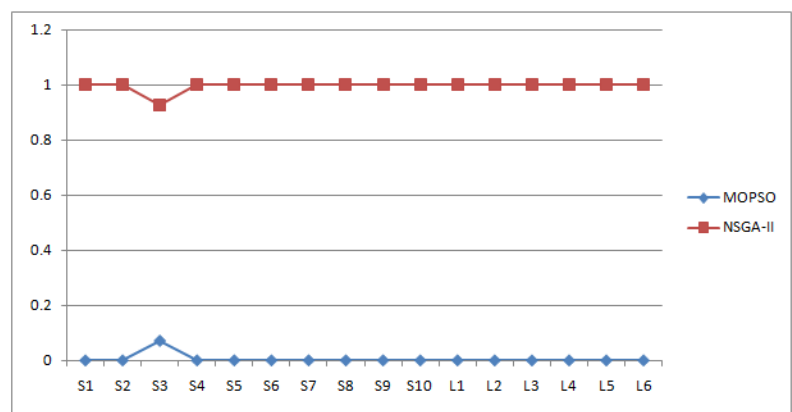

Fig. 2. The quality of Pareto solutions 


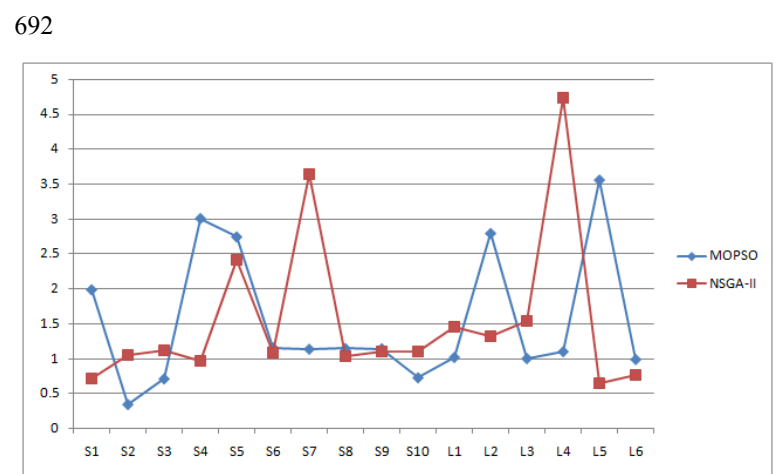

Fig. 3. Standard deviation of the Pareto solutions

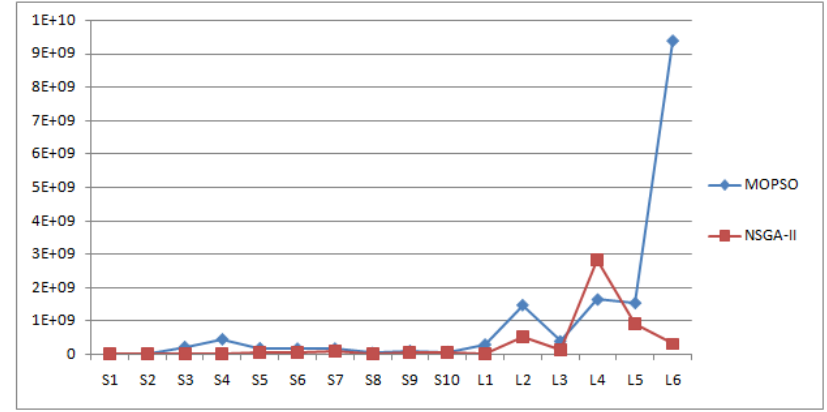

Fig. 4. Diversity of the Pareto solutions

On the contrary, NSGA-II preserves superior results against MOPSO. As we can observe from the results of Fig. 3, there is not much difference between the results of MOPSO and NSGA-II. According to the results of Fig. 4, MOPSO seems to provide more diverse solutions compared with NSGA-II. Fig. 5 shows that the proposed MOPSO needs less amount of time to reach Pareto solutions compared with NSGA-II. Finally, Fig. 6 presents a comparison between the Pareto solutions generated by both methods of NSGA-II and MOPSO algorithm.

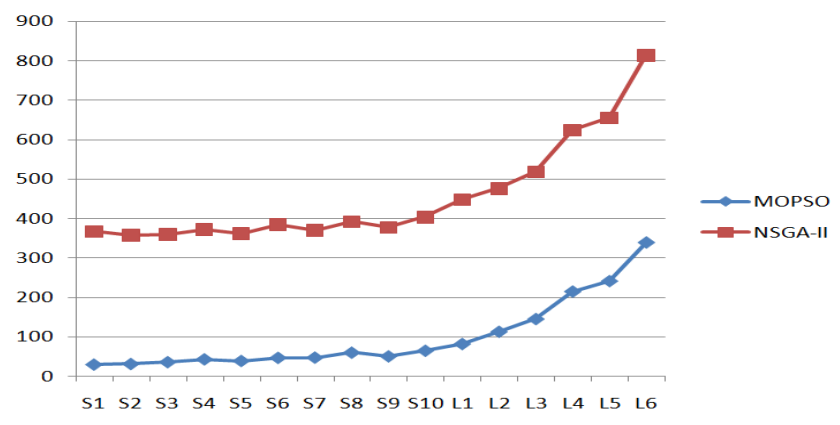

Fig. 5. CPU time (Sec.) of the Pareto solutions

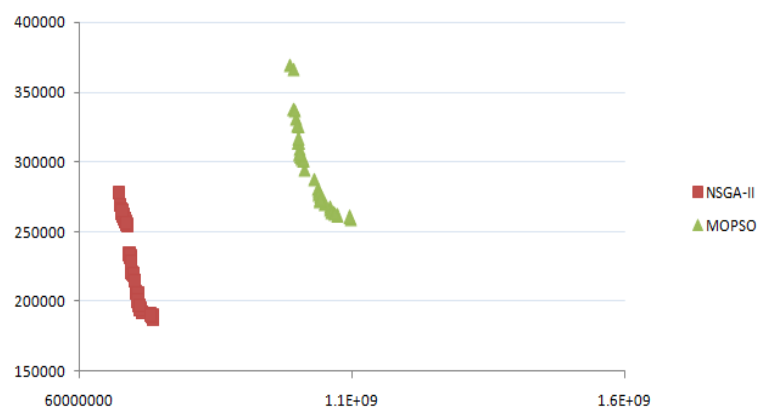

Fig. 6. Comparison of the Pareto solutions

\section{Conclusion}

In this paper, we have presented a multi-stage supply chain network by considering multi products, single resource and deterministic cost and demand. The proposed model of this paper has been formulated as a mixed integer programming and we have presented two metaheuristics namely MOPSO and NSGA-II to solve the resulted problems. The performance of the proposed models of this paper has been examined some randomly generated problem and the results have been discussed. The results has indicated while MOPSO has been able to generate more Pareto solutions in relatively less amount of time but NSGA-II has been able to provide better quality results. The comparison of the performance of two algorithms shown in Fig. and Fig. 6 shows that NSGA-II generates better quality solutions. The main reason is that it searches more solutions in feasible region.

As future study, we may consider uncertain data in cost, demand and such a model can be formulated in the context of robust optimization, and we leave it for interested researchers as future works.

\section{Acknowledgment}

The authors would like to thank the anonymous referees for their constructive comments on earlier version of this work. 


\section{References}

Altiparmak, F., Gen, M., Lin, L., \& Karaoglan, I. (2009). A steady-state genetic algorithm for multiproduct supply chain network design. Computers \& Industrial Engineering, 56(2), 521-537.

Altiparmak, F., Gen, M., Lin, L., \& Paksoy, T. (2006). A genetic algorithm approach for multiobjective optimization of supply chain networks. Computers \& Industrial Engineering. 51, 196-215.

Beamon, B. M. (1998). Supply chain design and analysis: Models and Methods International Journal of Production Economics. 55, 281-294.

Bottani, E., \& Rizzi, A. (2006). Strategic management of logistics service: A fuzzy QFD approach. International Journal of Production Economics. 103, 585-599.

Cardona-Valdés, Y., Álvarez, A., \& Ozdemir, D. (2010). A bi-objective supply chain design problem with uncertainty. Transportation Research Part C, 19, 821-823.

Chan, F. T. S., \& Kumar, N. (2009). Effective allocation of customers to distribution centres: A multiple ant colony optimization approach. Robotics and Computer-Integrated Manufacturing. 25, $1-12$.

Chan, F. T. S., Chung, S. H., \&Wadhwa, S. (2005). A hybrid genetic algorithm for production and distribution.Omega. 33, 345-355.

Chen, Y.M., \& Wang, W.S. (2010). A particle swarm approach to solve environmental/economic dispatch problem. International Journal of Industrial Engineering Computations, 1(1), 157-172.

CoelloCoello, C. A., Pulido, G. T., \& Lechuga, M. S. (2004). Handliny multiple objectives with particle swarm optimization. IEEE Transactions on Evolutionary Computation. 8, 256-279.

CoelloCoello, C. A.Aamont, G.B., \& Van Veldhuizen, D.A. (2007).Evolutionary Algorithms for Solving Multi-Objective Problems.SpringerScience+Business Media.ISBN 978-0-387-33254-3.

Das, S., Abraham, A., \& Konar, A. (2008). Particle swarm optimization and differential evolution Algorithms. Technical Analysis, Applications and Hybridization Perspectives. Studies in Computational Intelligence, 116, 1-38.

Deb, K., Pratap, A., Agarwal, S., \& Meyarivan, T. (2002). A Fast and Elitist Multiobjective Genetic Algorithm: NSGA-II. IEEE Transactions on Evolutionary Computation. 6, 182-197.

Du, F., \& Evans, G. W. (2008). A bi-objective reverse logistics network analysis for post-sale service. Computers \& Operations Research, 35, 2617-2634.

Erol, I., Ferrell Jr, W. G. (2004). A methodology to support decision making across the supply chain of an industrial distributor. International Journal of Production Economics. 89, 119-129.

Gen, M., \& Syarif, A. (2005). Hybrid genetic algorithm for multi-time period production/distribution planning. Computers \& Industrial Engineering. 48, 799-809.

Guillén, G., Mele, F.D., Bagajewicz, M.J., Espuña, A., \& Puigjaner, L. (2005). Multiobjective supply chain design under uncertainty. Chemical Engineering Science. 60, 1535-1553.

Guliashki, V., Toshev, H., \& Korsemov, C. (2009). Survey of Evolutionary Algorithms Used in Multiobjective Optimization. Institute of Information Technologies, 1113 Sofia.

Lee, Y. H., \& Kim, S. H. (2002). Production-distribution planning in supply chain considering capacity constraints. Computer \& Industrial Engineering. 43, 169-190.

Papageorgiou, L. G. (2009). Supply chain optimisation for the process industries: Advances and opportunities. Computers and Chemical Engineering, 33, 1931-1938.

Perea-López, E., Ydstie, B.E., \& Grossmann, I.E. (2003). A model predictive control strategy for supply chain optimization. Computers and Chemical Engineering, 27, 1201-1218.

Pishvaee, M. S., Zanjirani Farahani, R., \& Dullaert, W. (2010). A memetic algorithm for bi-objective integrated forward/reverse logistics network design. Computers \& Operations Research, 37, 11001112.

Poli, R., Kennedy, J., \& Blackwell, T. (2007). Particle swarm optimization: An overview. Swarm Intelligence. 1, 33-57.

Reyes-Sierra, M., \& CoelloCoello, C. A. (2006). Muti-objective particle swarm optimizers: A survey of the state-of-the-Art. International Journal of Computational Intelligence Research. 2, 287-308. 
Sabri, E. H., \& Beamon, B. M. (2000). A multi-objective approach to simultaneous strategic and operational planning in supply chain design. Omega. 28, 581-598.

Sedighizadeh, D., \& Masehian, E. (2009). Particle swarm optimization methods, taxonomy and applications. International Journal of Computer Theory and Engineering, 1, 1793-8201.

Sharaf, A. M., \& El.Gammal, A. A. (2009). A multi objective multi-stage particle swarm optimization MOPSO search scheme for power quality and loss reduction on radial distribution system. International Conference on Renewable Energies and Power Quality (ICREPQ'09).

Taghavi-fard, M.T., Javanshir, H., Roueintan, M.A., \& Soleimany, E. (2011). Multi-objective group scheduling with learning effect in the cellular manufacturing system. International Journal of Industrial Engineering Computations, 2, 617-630.

Tuzkaya, U.R., \& Önüt, S. (2009). A holonic approach based integration methodology for transportation and warehousing functions of the supply network. Computers \& Industrial Engineering, 56, 708-723.

Voudouris, V.T. \& Consulting, A. (1996). Mathematical programming techniques to debottleneck the supply chain of fine chemicals. Computers \& Chemical Engineering, 20S, S1269-S1274.

Xu, J., Liu, Q., \& Wang, R. (2008). A class of multi-objective supply chain network optimal model under random fuzzy environment and its application to the industry of Chinese Liquor. Information Sciences. 178, 2022-2043.

You, F., \& Grossmann, E. (2008). Design of responsive supply chain under demand uncertainty. Computers \& Chemical Engineering. 32, 3090-3111.

Zanjirani Farahani, R., \& Elahipanah, M. (2008). A genetic algorithm to optimize the total cost and service level for just-in-time distribution in a supply chain. International Journal of production Economics. 111, 229-243.

Zhou, G., Min, H., \& Gen, M. (2002). The balanced allocation of customers to multiple distribution centers in the supply chain network: a genetic algorithm approach. Computers \& Industrial Engineering, 43, 251-261. 